\title{
Adsorption and charge transfer interactions of bi-isonicotinic acid on $\mathbf{A g}(111)$
}

\author{
Robert H. Temperton, ${ }^{1}$ Andrew J. Gibson, ${ }^{1}$ Karsten Handrup, ${ }^{2}$ and James N. O'Shea ${ }^{1, a)}$ \\ 1) School of Physics, University of Nottingham, NG7 2RD, UK \\ 2) Synchrotron Radiation Research, Department of Physics, Lund University, Box 118, SE-221 00 Lund, \\ Sweden
}

The adsorption and charge transfer dynamics of the organic molecule bi-isonicotinic acid (4,4-dicarboxy2,2-bipyridine) on single crystal $\mathrm{Ag}(111)$ has been studied using synchrotron radiation-based photoemission, $\mathrm{x}$-ray absorption and resonant core spectroscopies. Measurements for multilayer and monolayer coverage are used to determine the nature of the molecule-surface interactions and the molecular orientation. An experimental density of states for the monolayer with respect to the underlying metal surface is obtained by combining x-ray absorption spectroscopy at the $\mathrm{N} 1 s$ edge and valence photoemission to measure the unoccupied and occupied valence states, respectively. This shows that the lowest unoccupied molecular orbital in the core-excited state lies energetically below the Fermi level of the surface allowing charge transfer from the metal into this orbital. Resonant photoelectron spectroscopy was used to probe this charge transfer in the context of super-spectator and super-Auger electron transitions. The results presented provide a novel interpretation of resonant core-level spectroscopy to explore ultra-fast charge transfer between an adsorbed organic molecule and a metal surface through the observation of electrons from the metal surface playing a direct role in the core-hole decay of the core-excited molecule.

\section{INTRODUCTION}

Understanding the charge transfer of electrons between molecules and surfaces is of critical importance to understanding the operation of molecular devices such as dyesensitised solar cells, ${ }^{[12]}$ water splitting photoelectrochemical cells, $\stackrel{3}{3}$ and a wide range of molecular electronics. The charge transfer dynamics from a molecule to a surface has been probed on the low femtosecond (fs) timescale using the core-hole clock implementation of resonant photoemission (RPES), which uses the core-hole decay channels to determine the degree of localization of a resonantly excited core-electron in an unoccupied valence state compared to the core-hole lifetime (typically a few fs). The underlying principle of this technique is to measure the electrons emitted from the core-hole decay process in which the originally excited electron is a direct participant. Charge transfer on the timescale of the core-hole lifetime is then inferred by the depletion of this core-hole decay channel. Charge transfer in the other direction, from the surface to the molecule, can be probed by a similar approach, where the electrons emitted from the corehole decay process in which the electron transferred from the surface into the lowest unoccupied molecular orbital (LUMO) of the core-excited molecule are measured. We have previously studied this phenomenon, $\frac{4 \sqrt{6}}{6}$ attributing the observed features in the resonant core spectroscopy to either super-Auger or super-spectator decay, ${ }^{[5}$ where electrons that have transferred from a surface into the LUMO are directly involved in the Auger-like core-hole decay, with or without a simultaneous transfer of a coreexcited electron into the empty density of states of the surface above the Fermi level, respectively. Molecules studied using this method range from fullerenes, $\frac{5}{5}$ wich

a) Electronic mail: J.Oshea@nottingham.ac.uk can be used in organic photovoltaic devices as an electron acceptor, ${ }^{7}$ to bi-isonicotinic acid,, 4 the ligand of the "N3" molecule used in dye sensitised solar cells. ${ }^{8} \mathrm{Al}-$ though bi-isonicotinic acid is largely responsible for the bonding between $\mathrm{N} 3$ and the titanium dioxide surface in a dye-sensitised solar cell ${ }^{1}$ and does itself show evidence of charge transfer between the molecule and a gold surface in both directions, ${ }^{4} \mathrm{~N} 3$ has been shown not to exhibit any super-spectator/super-Auger decay on the same surface. This has been attributed to the three-dimensional geometry of the N3 organometallic complex in which the bi-isonicotinic acid ligands coordinate the metal centre orthogonal to one other so that the delocalised $\pi$ orbtials cannot interact with the surface, while the bi-isonicotinc acid molecule on its own can maximise this interaction of the unfilled LUMO with filled metal states by adsorbing parallel to the surface. ${ }^{6}$

In these examples of super-spectator/super-Auger decay, $\frac{415}{4}$ electrons are able to transfer from the metal into the molecule because the LUMO of the core-excited molecule partially overlaps energetically with the Fermi level of the metal surface in the core excited state. This study examines the system of bi-isonicotinic acid on a $\operatorname{Ag}(111)$ surface, where the LUMO lies entirely below the Fermi level (in the core excited state) which could, in principle, increase the number of electrons transferred and made available to participate in the superspectator/super-Auger decay.

\section{METHOD}

Measurements were carried out at the I311 beamlime ${ }^{9}$ on the MAX-II storage ring at $M A X-L a b$, Sweden. This undulator-based beamline has an energy range of $30-$ $1500 \mathrm{eV}$ and was configured to give an energy resolution of $5 \mathrm{meV}$ to $150 \mathrm{meV}$ between $50 \mathrm{eV}$ and $600 \mathrm{eV}$. The 
a)
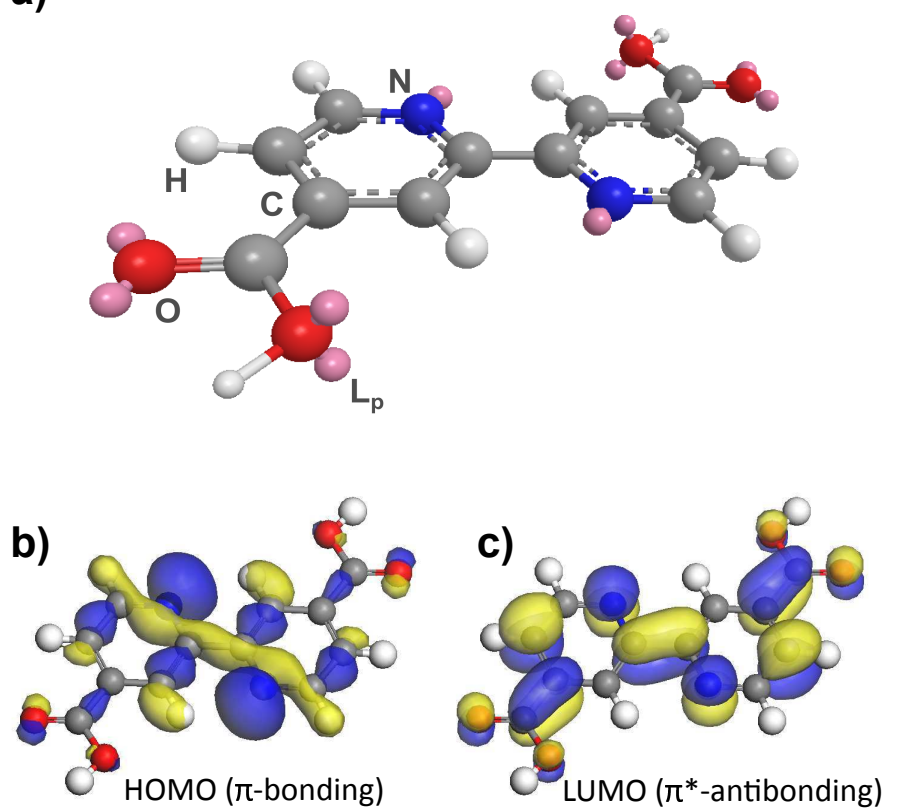

FIG. 1: Bi-isonicotinic acid

(4,4-dicarboxy-2,2-bipyridine). a) Minimised structure of the gas-phase molecule in the ground state, unmodified by any potential effects of the core-hole created in the core-excited state. Gray, blue, red and white balls represent carbon, nitrogen, oxygen and hydrogen atoms respectively. a) Lone pair electrons $\left(L_{p}\right)$ are indicated in pink. b) and c) Spatial distribution of the HOMO and LUMO orbitals of the ground state molecule with yellow and blue lobes representing the orbital phases (Geometry and orbitals simulated using the DMol3 package in Materials Studio, Accelrys, using a GGA PBE functional).

light produced by this beamline was highly elliptically polarized to the extent the polarization was considered linear. The spot of light incident onto the sample had an approximate size of $0.5 \mathrm{~mm} \times 0.1 \mathrm{~mm}$. The end station was equipped with an upgraded Scienta SES200 hemispherical electron analyser where a straight slit with width $0.8 \mathrm{~mm}$ was used giving an analyser resolution of $10 \mathrm{meV}$ and $100 \mathrm{meV}$ for pass energies $20 \mathrm{eV}$ and $50 \mathrm{eV}$ respectively. The base pressures in the preparation and analysis chambers were $1 \times 10^{-9} \mathrm{mbar}$ and $1 \times 10^{-10} \mathrm{mbar}$, respectively. A single crystal of $\mathrm{Ag}(111)$ was mounted on a loop tungsten wire that enabled resistive heating of the sample and a thermocouple junction was spot welded to the side of crystal allowing temperature measurement. The crystal was cleaned using $1 \mathrm{kV} \mathrm{Ar}^{+}$ion sputtering and $\sim 550^{\circ} \mathrm{C}$ anneal cycles until there was no $\mathrm{C} 1 \mathrm{~s}$ peak visible in the XPS spectra and the $\mathrm{Ag} 3 d$ peaks valence structure had their respective characteristic line shapes.

Bi-isonicotinic acid (4,4-dicarboxy-2,2-bipyridine from Alfa Aesar), shown in Figure 1, was deposited onto the surface in-situ by sublimation from a Knudsen-type cell evaporation source. Low coverage and high coverage films (controlled by exposure time) were measured using x-ray photoelectron spectroscopy (XPS), x-ray absorption spectroscopy (XAS) spectroscopy, and resonant photoelectron spectroscopy (RPES). XPS, measured with an undulator gap of $17 \mathrm{~mm}$, was calibrated to the binding energy scale by aligning the silver Fermi edge to $0 \mathrm{eV}$, giving an uncertainty in the binding energy scale on the order of $0.05 \mathrm{eV}$. The data presented has been processed by the subtraction of a Shirley background and peak fitting has been performed using a pseudo-Voigt function where a combination of Gaussian and Lorentzian lineshapes were fitted to the data. The Lorentzian width was fixed at $0.3 \mathrm{eV}$ and the Gaussian contribution allowed to vary in the fitting procedure.

XAS and RPES were measured over the N $1 s$ absorption edge with a taper applied to the undulator to minimise variations in photon flux at different energies. The photon energy was calibrated using the kinetic energy difference between Ag $3 d$ XPS peaks collected using first and second order light from the monochromator. The spectrometer was operated in fixed constant final state and constant initial state modes for XAS and RPES measurements respectively and swept mode for XPS. Given the long scan durations (up to two hours), the sample was continually moved during measurements at a rate determined to be safe by beam-damage studies.

This paper refers to monolayer and multilayer coverages - these two datasets correspond to two separate depositions. For the multilayer films, the $\mathrm{Ag} 3 d$ substrate peaks were almost undetectable in the XPS measurements. For the monolayer film, molecules were deposited until the peak ratios in the XPS and the lineshape of the valence band region were consistent with monolayer and sub-monolayer data for these molecules

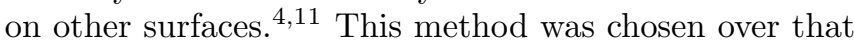
of annealing a multilayer ${ }^{411}$ in order to avoid the potential damage of the molecules through excessive heating as previously observed in the $\mathrm{N} 1 s$ XAS for bi-isonicotinic acid on the $\mathrm{Au}(111)$ surface!

\section{RESULTS AND DISCUSSION}

The photoemission spectra provide an indication of how the bi-isonicotinic acid molecules interact with the surface by comparing the monolayer film, where molecules can interact with the surface, and multilayer where the molecules able to interact with the surface are deeply buried so are not measurable using photoemission. The most dramatic difference between the two is seen in the $\mathrm{O} 1 s$ spectra, shown in Figure 2. The multilayer data is shown in Figure 2 a exhibiting two overlapping peaks at $533.6 \mathrm{eV}$ and $532.4 \mathrm{eV}$. These are attributed to the oxygen atoms in the hydroxyl $(\mathrm{C}-\mathrm{OH})$ and carbonyl $(\mathrm{C}=\mathrm{O})$ groups in the carboxylic acid group of the molecule, respectively. ${ }^{11}$ For the monolayer coverage we 
a)

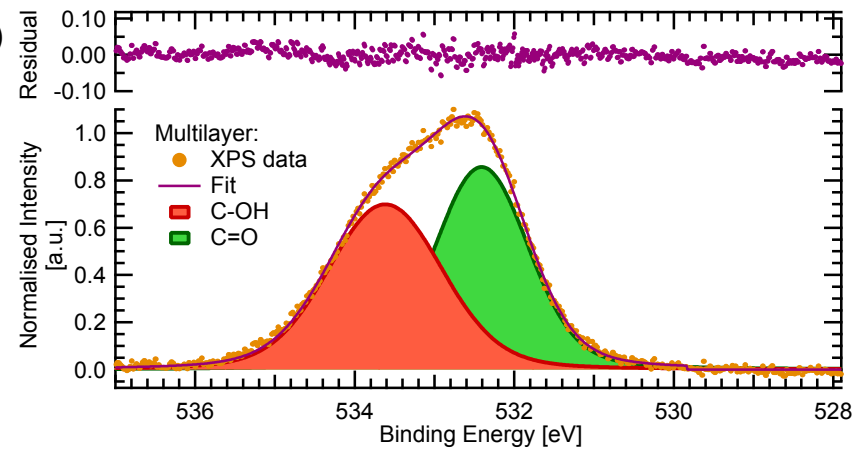

b)

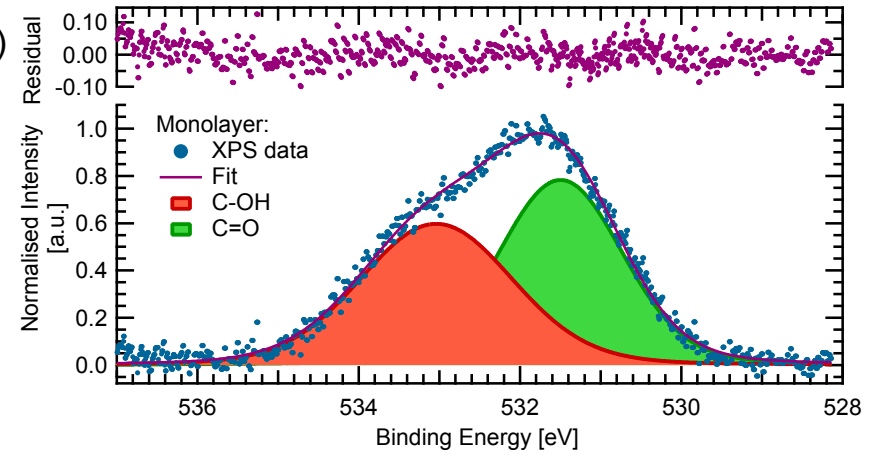

FIG. 2: O $1 s$ XPS spectra for a) multilayer and b) monolayer films of bi-isonicotinic acid on $\mathrm{Ag}(111)$. The data has had a Shirley background subtracted. The curve fitting reveals two peaks which we attribute to the carbonyl and hydroxyl oxygen atoms (residual shown above each spectrum). The area under both peaks is equal. The thick film data was collected at normal incidence with a photon energy of $722 \mathrm{eV}$. The thin film data was collected at a grazing angle of $55^{\circ}$ at $600 \mathrm{eV}$ to enhance surface sensitivity.

also find two peaks, here at $533.0 \mathrm{eV}$ and $531.5 \mathrm{eV}$, attributed as for the multilayer. The two peaks each have a normalised area of 0.5 , consistent with the stoichiometry of the molecule (a 1:1 ratio of oxygen atoms in the two chemical environments).

There are a few notable features of the $\mathrm{O} 1 \mathrm{~s}$ spectra. First, the position of the peaks has changed between the monolayer and multilayer coverages - there is a general shift towards lower binding energy for the monolayer. Such shifts are commonly attributed to screening of the core-hole by electrons in the metal surface ${ }^{[12}$ but we would expect this shift to be uniform across the entire spectrum. Here we see that the carbonyl peak has shifted relative to the hydroxyl peak by $0.35 \mathrm{eV}$ indicative of a stronger interaction between of carbonyl group with the surface compared to the hydroxyl group. Secondly, the peaks are broader in the monolayer data, most likely due to a number of different possible bonding/interaction sites on the surface.

C $1 s$ XPS data is shown in Figure 3 for the multilayer and monolayer. There are two clear peaks visible at approximately $286 \mathrm{eV}$ and $289.5 \mathrm{eV}$ and a small peak

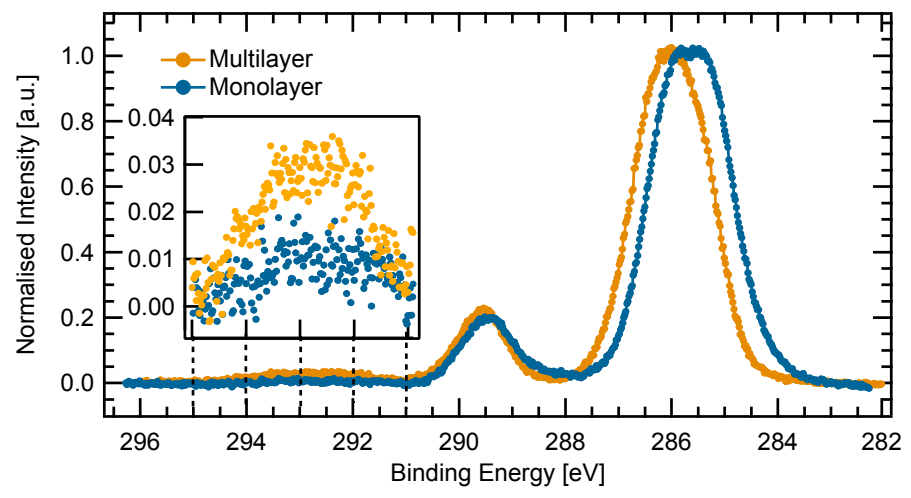

FIG. 3: C $1 s$ XPS spectra for monolayer and multilayer coverage of bi-isonicotinic acid on $\operatorname{Ag}(111)$. The peak at $289.5 \mathrm{eV}$ is attributed to the carboxylic acid group and the peak at $\sim 286 \mathrm{eV}$ to the pyridine ring. A Shirley background was subtracted from both spectra. An excitation energy of $514 \mathrm{eV}$ and $340 \mathrm{eV}$ was used for the multilayer and monolayer, respectively.

at $\sim 293 \mathrm{eV}$. The latter is shown magnified in the inset. As with previous studies, $\frac{11}{11}$ we have assigned the lower binding energy peak $(\sim 268 \mathrm{eV})$ to the carbon atoms in the pyridine ring and the higher binding energy peak $(\sim 289.5 \mathrm{eV})$ to the carboxylic group. The low intensity satellite at $\sim 293 \mathrm{eV}$ is attributed to a shakeup feature. ${ }^{13}$ For the pyridine carbon, there is a clear shift of $\sim 0.35 \mathrm{eV}$ to lower binding energy. As discussed above in the context of the $\mathrm{O} 1 s$ spectra, such a shift can be explained by screening effects. As before, we would expect an equal shift of the entire region - screening alone therefore fails to explain why the shift is only apparent for the pyridine ring and not the carboxyl carbon atoms. Given the $\mathrm{O} 1 \mathrm{~s}$ data where the carbonyl oxygen is potentially interacting with the surface, we propose that there may be a slight positive charge formed at the carboxyl carbon atom due to the surface interaction drawing electron density away from it. This increases the binding energy of the carboxylic $\mathrm{C} 1 s$ featuring countering the negative binding energy shift caused by screening from the surface.

Taking the oxygen and carbon spectra into consideration, we can rule out the possibility that the silver is causing deprotonation of the carbonyl group. If deprotonation occurs, there are two possible scenarios. Firstly, for the case of partial deprotonation of the monolayer molecules, we would expect a third, lower binding energy peak in the $\mathrm{O} 1 s$ and a further peak in the $\mathrm{C} 1 s$ region close to the carboxylic group binding energy. Cebula et $a i^{14}$ have recently studied similar small organic molecules featuring carboxylic acid groups and have seen this behaviour when using electrochemical underpotential deposition. While their XPS findings may not translate directly to the present study (where the deposition technique does not create ionised species by design), the comparison is still of value. For the second scenario, where all the carboxylic groups are deprotonated, we would only 


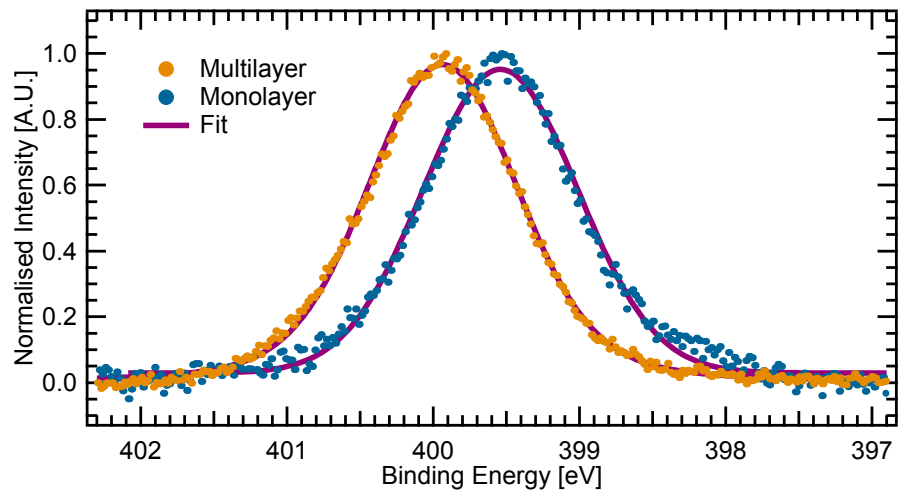

FIG. 4: N $1 s$ XPS spectra for multilayer and monolayer coverages of bi-isonicotinic acid on $\mathrm{Ag}(111)$, fitted using identical parameters. Both spectra were measured at normal emission with an excitation energy of $\sim 500 \mathrm{eV}$

and have had a Shirley background subtracted.

see two peaks in the $\mathrm{O} 1 \mathrm{~s}$, corresponding to $\mathrm{C}=\mathrm{O}$ and $\mathrm{C}$ $\mathrm{O}^{-}$, although in practice the binding energies of these two environments are indistinguishable in the $\mathrm{O} 1 \mathrm{~s}$ and instead are typically observed as a single peak at the $\mathrm{C}=\mathrm{O}$ binding energy. The $\mathrm{C} 1 s$ would also show two peaks but with the carboxylic group shifted to lower binding energy.

Here, we do not see any evidence of deprotonation in the $\mathrm{C} 1 s$ spectra. Li et a $\sqrt{15}$ have also studied isonicotinic acid (half of the bi-isonicotinic acid molecule), deposited via sublimation from a K-cell, on $\mathrm{Ag}(111)$ and also measured $\mathrm{O} 1 s$ XPS data consistent with no deprotonation of the monolayer. We can also rule out the possibility that the data may contain a multilayer contribution - attempting to include a second set of peaks into the curve fitting in a way that would be consistent does not lead to a realistic curve-fit. The difference between the monolayer and multilayer data therefore likely represents real shifts in binding energy between the bulk molecule and the adsorbed molecules due to the combined effect of screening and the interaction of the carbonyl moiety with the $\operatorname{Ag}(111)$ surface.

The corresponding N $1 s$ XPS spectra are shown in Figure 4 exhibiting a single peak that is the same shape for the monolayer and multilayer films, which is consistent with the single bonding environment exhibited by nitrogen atoms. There was a small shift, again consistent with screening effects, between the thin and thick film measurements of $0.38 \mathrm{eV}$ (compared to screening shifts of $0.35 \mathrm{eV}$ seen in both the $\mathrm{O} 1 s$ and $\mathrm{C} 1 s$ spectra).

Previous studies have considered the effects of hydrogen bonding between bi-isonicotinic acid molecules (and related pyridine carboxylic acid molecules) and have seen little evidence indicative of hydrogen bonding between biisonicotinic acid molecules. $\frac{16}{16}$ This is consistent with what we see here - there are no shifts or multiple peaks in $\mathrm{N}$ $1 s$ or $\mathrm{O} 1 s$ that would indicate the presence of $\mathrm{N} \cdots \mathrm{O}-\mathrm{H}$ hydrogen bonding. The weak feature at $\sim 398.5$ in the

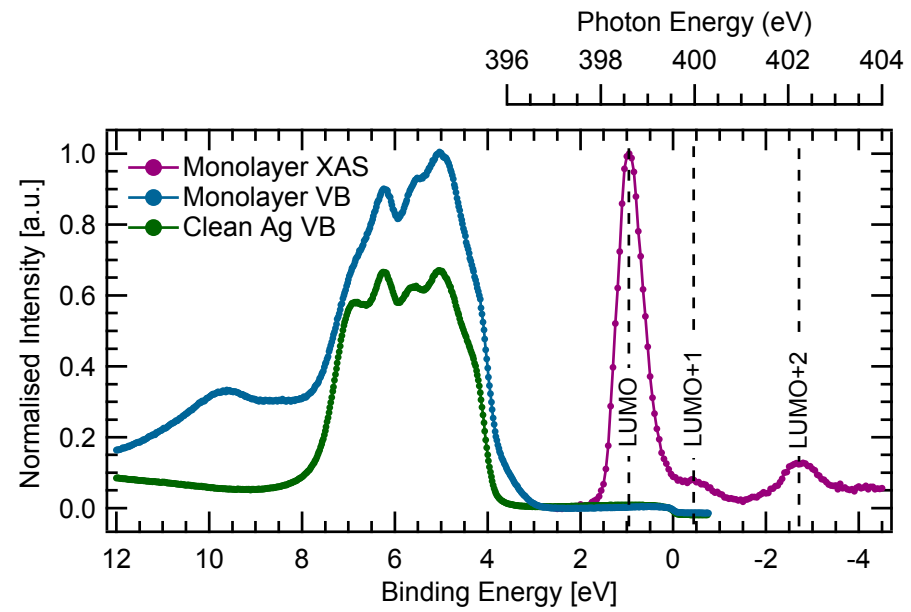

FIG. 5: Density of states plot of a monolayer of bi-isonicotinic acid on $\mathrm{Ag}(111)$ measured at normal emission. The purple line shows an x-ray absorption spectra (measured over the N $1 s$ absorption edge) showing unoccupied states placed on a binding energy

scale. The blue and green lines shows the valence spectrum of the monolayer and clean surface respectively, measured by photoemission spectroscopy with a photon energy of $50 \mathrm{eV}$. All spectra were collected at normal emission.

monolayer $\mathrm{N} 1 s$ is on the wrong side of the peak to be attributed to hydrogen bonding. The origin of this weak feature is still undetermined.

An experimental density of states for the monolayer is presented in Figure 5. This comprises the $\mathrm{N} 1 s$ XAS placed on a binding energy scale by subtracting the calibrated absorption energy from the measured N 1s XPS binding energy calibrated to the Fermi level. The valence band photoemission of the monolayer (also calibrated to the Fermi level) measured at $50 \mathrm{eV}$ is placed on the same binding energy scale along with the same spectrum measured for the clean silver surface. In this way we can get a complete picture of the energetic overlap of the occupied and unoccupied molecular orbitals with respect to the filled and empty states of the underlying surface. This method is discussed in further detail elsewhere. 10 We have assigned the peaks in the XAS at $B E \approx-0.5 \mathrm{eV}$ and $B E \approx-2.5 \mathrm{eV}$, as marked on the figure, to the LUMO+1 and LUMO+2 states of the core-excited molecules. The N $1 s$ was chosen due to the well-separated $\pi^{*}$ resonances and to allow comparison with previous studies of bi-isonicotinic acid on different surfaces. $\stackrel{46}{6}$

It is clear from Figure 5 that the LUMO, the largest peak in the XAS data $(B E=1 \mathrm{eV})$, lies entirely below the Fermi edge for the core-excited molecule. This, in principle, allows electrons to charge transfer from the sil- 

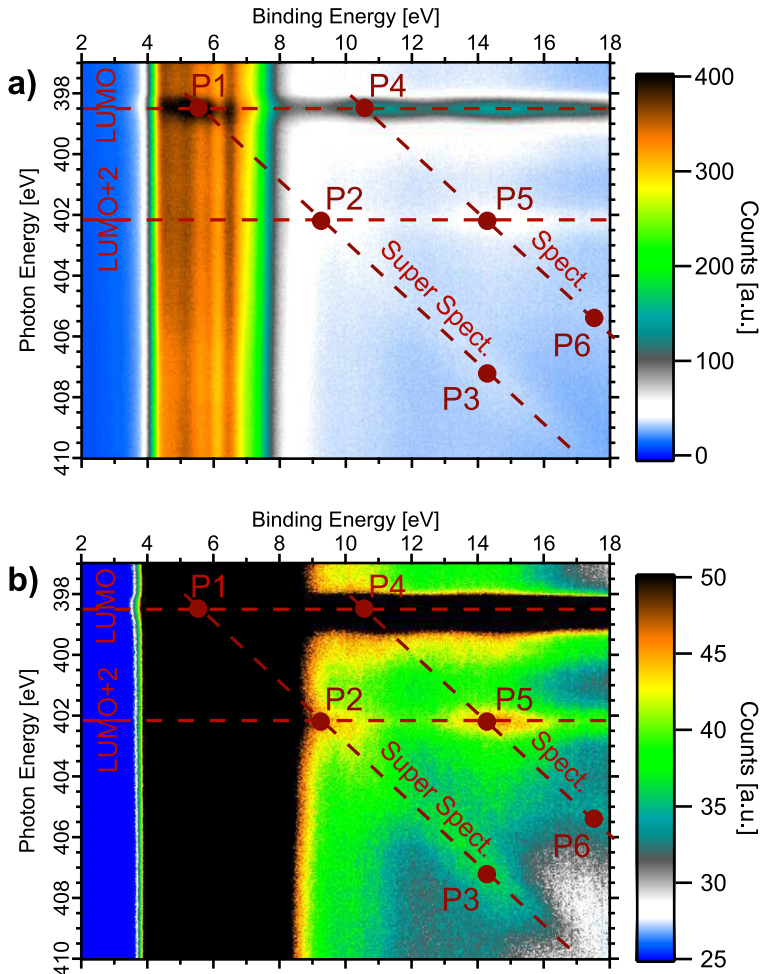

c)

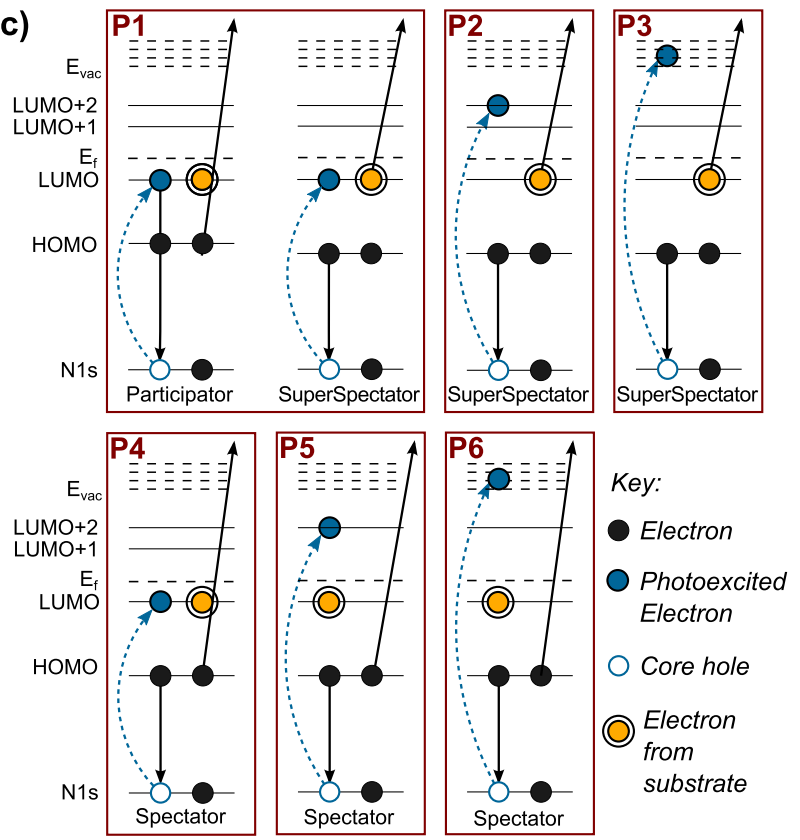

FIG. 6: Resonant photoemission map of a monolayer of bi-isonicotinic acid on $\mathrm{Ag}(111)$ collected at normal emission plotted using two colour map scales. The LUMO energy levels are indicated with horizontal dashed lines and the diagonal lines highlight constant kinetic energy features. Figure a) is scaled across the whole range of data and b) to emphasise the spectator and super-spectator features. Figure c) shows schematics of proposed decay mechanisms marked as points P1-P6 (on the RPES data) after excitation of an

$\mathrm{N} 1 s$ core electron (shown with a dotted arrow). ver surface into the LUMO of the core-excited molecule. It is worth noting that this partially filled state is likely to cause slight shifts in the band positioning, and critically, these electrons are able to participate in the relaxation of the molecule back to the ground state after the coreexcitation, a process that can be explored in detail using resonant photoemission spectroscopy (RPES).

The resonant photoemission data is shown in Figures 6 and 7 . The 2D plots show the intensity of emitted valence photoelectrons as a function of both incident photon energy and their measured binding energy. Sweeping through a range of photon energies finds points where the incident light is on resonance with an excitation process (the photon energy matches the energy difference between a core level and a molecular orbital) which is measured by an increased flux of emitted photoelectrons. Measuring the binding energy of these emitted electrons allows us to infer the decay channel by which the system relaxed back to its final state.

Figure 6 was collected with the surface was oriented normal to the analyser (normal emission)and Figure 7 with the surface normal to the incident light $\left(55^{\circ}\right.$ emission angle). The horizontal dashed lines mark the regions of intensity that appear at constant excitation energy - we have attributed these to excitation into LUMO states. The LUMO+1 level is not marked as the intensity of features attributed to the $\mathrm{LUMO}+1$ are too weak (see $\mathrm{N} 1 s$ XAS in Figure 5) to be distinguished from the high-photon energy tail of the LUMO. The diagonal lines highlight constant kinetic energy features that we have attributed to spectator and super-spectator/super-Auger decay channels. All of these dashed lines are shown in the same places on all the plots. Six key points of interest on these lines (P1-P6) are marked each with associated schematic diagrams at the bottom of the panel (Figure 6c). These describe possible decay mechanisms that give rise to the different spectral features, shown with solid arrows, after the initial excitation (shown with dashed arrows) and creation of the core-hole.

The enhancement at P1 - located at the intersection of the HOMO binding energy and the LUMO absorption energy - is due to participator electrons. This is a decay mechanism where the originally photoexcited electron recombines with the core-hole and another electron from the HOMO is emitted. For spectator decay (P4, $\mathrm{P} 5$ and $\mathrm{P} 6$ ) on the other hand, a core electron is excited into an unoccupied molecular orbital and does not further participate in the relaxation of the system into its final state (within the lifetime of the core-hole). Electrons from occupied molecular orbitals undergo an Auger-like decay process where one electron recombines with the core-hole and another is emitted into the vacuum. The kinetic energy of these electrons is constant and must be lower than participator electrons by at least the HOMOLUMO gap. The intensity around points $\mathrm{P} 4$ and P5 on the spectator decay line result from when the incident energy is on resonance with transitions between the $\mathrm{N} 1 s$ core level and the LUMO and LUMO+2 states respec- 

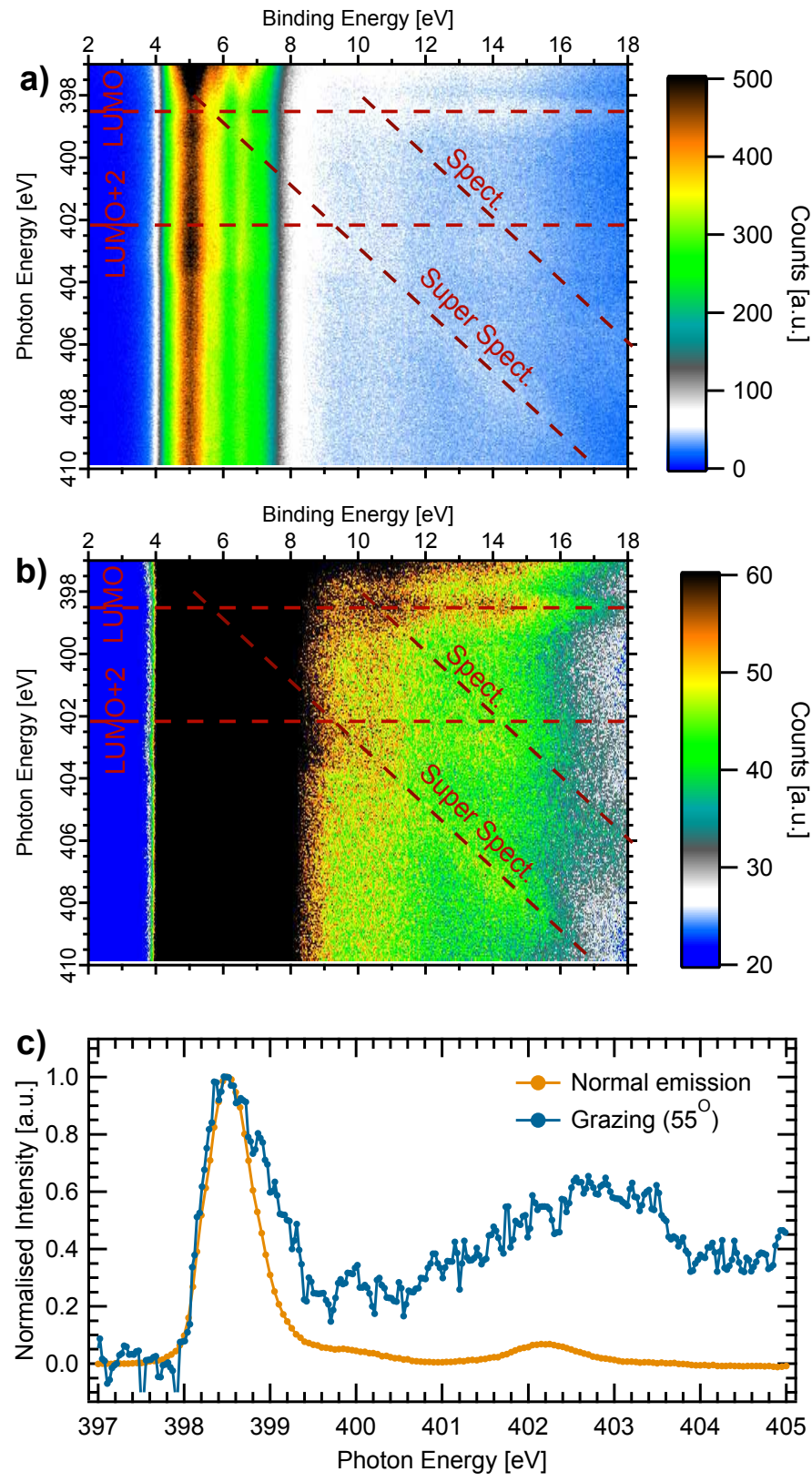

FIG. 7: Resonant photoemission scan of a monolayer of bi-isonicotinic acid on $\mathrm{Ag}(111)$ collected at a grazing angle of $55^{\circ}$ from normal emission using two colour map scales. The LUMO energy levels are indicated with horizontal dashed lines and the diagonal lines highlight constant kinetic energy features. Figure a) is scaled across the whole range of data and b) to emphasise the spectator and super-spectator features. Figure c) compares XAS extracted from both this grazing incidence RPES and the normal emission RPES shown in Figure 6 tively. P6 shows the excitation of the core electron into the continuum of states above the vacuum level (including sigma anti-bonding orbitals, $\sigma^{*}$ ). This again decays via the spectator channel as shown in Figure 6r.

The LUMO of the core-excited molecule lies below the Fermi level due (in part if not entirely) to the effect of the core-valence exciton generated in the x-ray absorption process increasing the binding energy of the measured unoccupied orbital. Since the XAS process necessarily measures the energy of the LUMO in the presence of a corehole, techniques such as two photon photoemission or inverse photoemission (where no core-hole is present) would be required to measure where the LUMO lies in the ground state. This is an important distinction since if the LUMO only overlaps with filled states in the surface while in the core-excited state, then charge transfer into this orbital must take place on the timescale of the core-hole lifetime (a few femtoseconds). Alternatively, if the LUMO lies at least to some extent below the Fermi level already in the ground state, then charge transfer need not be ultrafast. It is also worth noting that both scenarios are possible simultaneously, as the LUMO may lie partially below the Fermi level in the ground state and excitonically shifted further in energy in the core-excited state. A comparison of a similar experimental density of states for the N3 dye molecule (containing two bi-isonicotinic acid ligands) in the core-excited state with the optical gap of the molecule suggests an excitonic shift of around $1.6 \mathrm{eV} ! 1$ Such a shift in the bi-isonicotinic acid data shown in Figure 5 would imply that the LUMO lies entirely above the Fermi level in the ground-state, suggesting that any charge transfer into the core-excited LUMO would be need to be on the timescale of the core-hole lifetime.

Since the LUMO does in any case lie below the Fermi level in the core-excited state, electrons from the silver surface can partially fill this orbital opening up the potential for super-spectator and super-Auger core-hole decay channels. Super-spectator decay is again an Auger-like like process where the electrons in the LUMO (originally from the surface) along with an electron from an occupied state (in this case the HOMO), are involved in photoemission and recombination with the core-hole. P1, P2 and P3 show this mechanism after excitation of an N $1 s$ core electron into the LUMO, LUMO +2 and above the vacuum level, respectively. The electrons emitted via this process have constant kinetic energy and with a separation from spectator decay equal to the HOMOLUMO gap. Therefore at point $\mathrm{P} 1$, this process is indistinguishable from participator decay - the final state of both processes is the same and the emitted photoelectron will have the same energy.

A further possibility is a super-Auger decay process in which a photoexcited electron in an unoccupied state can 
charge transfer into the metal as well as an electron simultaneously transfering from the metal to the LUMO. The electron originally from the surface is emitted (as in super-spectator decay). The super-Auger mechanism is therefore an example of bi-directional charge transfer ${ }^{17}$ that can occur when the photoexcited electron is not localised to the unoccupied state within the core-hole lifetime. This mechanism is discussed in detail by Gibson et $a l$ in the context of $\mathrm{C}_{60}$ on single crystal metal surfaces. $\frac{18}{18}$ The conceptual picture of this core-hole decay mechanism can be visualised in Figure 6c by considering the originally excited electron (shown in blue) as having tunnelled away and plays no further role. This scheme is valid for panels P2 to P6 as these involve states that lie above the Fermi level and therefore overlap with empty states in the conduction band. For panel P1 however this is not the case and the illustrated picture with the originally excited electron localised in the LUMO represents the only available option.

The super-Auger model is consistent with two important observations in the RPES data. Firstly, the diagonal feature associated with this charge-transfer Auger process has a constant kinetic energy. This might imply the absence of a spectator shift arising from the localised electron in the unoccupied valence state, since such an effect should be weaker when the core-electron is excited to a state above the vacuum level, which lays just above the LUMO+2 absorption. For a true super-spectator process we might therefore expect a shift to lower kinetic energy when exciting into a localised state compared with excitation into a quasi-bound state. Secondly, as observed in Figure $6 \mathrm{a}$, there the participator feature is not symmetric around the point where the so-called super-spectator line intersects the LUMO absorption energy at point P1, but rather extends to higher kinetic energy (lower binding energy). Since ultra-fast bi-directional charge transfer is not possible for the LUMO (since it lies entirely below the Fermi level of the metal surface), only the participator and super-spectator processes illustrated in panel $\mathrm{P} 1$ of Figure 6r are valid. Both of these processes contain both the electron transferred from the surface and the originally excited electron in the LUMO and should therefore be shifted to slightly higher kinetic energy (the so-called spectator shift) than that of the super-Auger intersection at point P1. A super-Auger process is therefore the most likely origin of the high kinetic energy Auger-like feature observed for states above the Fermi level, while a superspectator process takes place for the LUMO, which lies entirely below the Fermi level.

Figure 7 shows the same experiment carried out at normal incidence, with the electric field vector of the light in the plane of the surface. Here, at the LUMO resonance photon energy there is very little intensity attributed to Auger and Auger-like features - this implies the molecules are laid flat on the surface as in this geometry the $\pi^{*}$ orbitals of the planar molecule have a low interaction crosssection with the electric field vector of the incident light. The $\sigma^{*}$ orbitals on the other hand lie in the same plane as the surface leading to spectator and super-spectator features being measurable in both orientations. This is further demonstrated in Figure $7 \mathrm{k}$ ) which compares the XAS extracted from the Auger region of both normal and grazing emission datasets. The two plots are normalised to the intensity of the LUMO peak.

The behaviour of bi-isonicotinic acid on $\mathrm{Ag}(111)$ as studied here is consistent with the work of Taylor et al. who have previously studied the same molecule on the similar $\mathrm{Au}(111)$ surface. ${ }^{4}$ They showed using angle resolved x-ray absorption measurements that the molecule is almost parallel to the surface (with the ring having an average angle greater than $70^{\circ}$ to the surface normal). On $\mathrm{Au}(111)$ as Taylor et al. demonstrated with their $\mathrm{N} 1 \mathrm{~s}$ XAS-derived experimental density of states, the LUMO only partially overlaps with the Fermi level of the gold substrate. On $\operatorname{Ag}(111)$ however, the entire state lies below the Fermi level providing a clear cut-off between the possibilities for super-spectator and super-Auger decay below and above the Fermi level, respectively.

In principle, the intensity of the super-Auger feature could be used to estimate the degree of electron transfer from the surface into the LUMO, but in practice there is no convenient point of normalisation that would allow comparison between different systems. However, in the case of $\mathrm{C}_{60}$ on $\mathrm{Ag}(111)$ reported recently, $\frac{18}{18}$ an additional super-Auger channel shifted to higher kinetic energy from the Core/LUMO/HOMO channel by the HOMO-LUMO gap. Such a Core/LUMO/LUMO decay process requires two electrons to be localised in the LUMO and is not observed in the present case. We should therefore conclude that there is no evidence that more than one electron is transferred from the metal surface into the LUMO of the core-excited molecule for bi-isonicotinic acid on $\mathrm{Ag}(111)$.

\section{CONCLUSIONS}

This study has presented high resolution XPS and XAS data for bi-isonicotinic acid deposited in-situ on a $\mathrm{Ag}(111)$ single crystal surface. The XPS suggests an interaction between the carboxyl group and the silver surface. The N $1 s$ XAS shows that the LUMO lies below the Fermi level therefore allowing the state to be partially occupied with electrons from the metal. Resonant photoemission measurements allowed us to probe this charge transfer via measurement of the super-spectator and super-Auger decay mechanisms whereby an electron originally from the metal is involved in the decay of the core excited state of the molecule. Due to the energetic alignment of the unoccupied molecular orbitals with respect to the density of states of the underlying surface, super-Auger decay arising from ultrafast bi-directional charge transfer dominates for states above the Fermi level, while super-spectator decay dom- 
inates for the LUMO state that lies below. The results presented provide a novel interpretation of resonant core-level spectroscopy to explore ultrafast charge transfer between an adsorbed organic molecule and a metal surface through the observation of electrons from the metal surface playing a direct role in the core-hole decay of the coreexcited molecule.

\section{ACKNOWLEDGEMENTS}

We would like to thank the authors of the Taylor et al study 4 for allowing us access to the RPES data presented in their 2007 paper. Funding was provided by RCUK - Engineering and Physical Sciences Research Council (EPSRC) and Molecularspray Ltd through a DTG/CASE conversion studentship. The research leading to these results has received funding from the European Community's Seventh Framework Programme (FP7/2007-2013) CALIPSO under grant agreement 312284 . We would also like to express our thanks to the staff at MAX-lab for their technical assistance, especially Dr. Karina Schulte for her support on beamline I311.

${ }^{1}$ L. C. Mayor, J. Ben Taylor, G. Magnano, A. Rienzo, C. J. Satterley, J. N. O'Shea, and J. Schnadt, J. Chem. Phys. 129, 114701 (2008).

${ }^{2}$ M. Weston, A. J. Britton, and J. N. O'Shea, J. Chem. Phys. 134, 054705 (2011)
${ }^{3}$ M. Weston, K. Handrup, T. J. Reade, N. R. Champness, and J. N. O'Shea, J. Chem. Phys. 137, 224706 (2012)

${ }^{4}$ J. B. Taylor, L. C. Mayor, J. C. Swarbrick, J. N. O'Shea, C. Isvoranu, and J. Schnadt, J. Chem. Phys. 127, 134707 (2007).

${ }^{5}$ A. J. Britton, A. Rienzo, J. N. O'Shea, and K. Schulte, J. Chem. Phys. 133, 094705 (2010)

${ }^{\circ}$ A. J. Britton, M. Weston, J. B. Taylor, A. Rienzo, L. C. Mayor, and J. N. O'Shea, J. Chem. Phys. 135, 164702 (2011)

${ }^{7}$ K. Schulze, C. Uhrich, R. Schüppel, K. Leo, M. Pfeiffer, E. Brier, E. Reinold, and P. Bäuerle, Adv. Mater. 18, 2872 (2006)

${ }^{8}$ M. Grätzel, J. Photoch. Photobio. C 4, 145 (2003)

${ }^{9}$ R. Nyholm, J. N. Andersen, U. Johansson, B. N. Jensen, and I. Lindau, Nucl. Instrum. Meth. A 467-468, 520 (2001)

${ }^{10}$ J. Schnadt, J. OShea, L. Patthey, J. Krempaský, N. Mårtensson, and P. Brühwiler, Phys. Rev. B 67, 235420 (2003)

${ }^{11}$ J. N. O'Shea, J. B. Taylor, L. C. Mayor, J. C. Swarbrick, and J. Schnadt, Surf. Sci. 602, 1693 (2008)

${ }^{12}$ M. Ruiz-Osés, D. G. De Oteyza, I. Fernández-Torrente, N. Gonzalez-Lakunza, P. M. Schmidt-Weber, T. Kampen, K. Hom, A. Gourdon, A. Arnau, and J. E. Ortega, ChemPhysChem 10, 896 (2009)

${ }^{13}$ C. Enkvist, S. Lunell, B. Sjögren, S. Svensson, P. A. Brühwiler, A. Nilsson, A. J. Maxwell, and N. Mårtensson, Phys. Rev. B 48, 14629 (1993)

${ }^{14}$ I. Cebula, H. Lu, M. Zharnikov, and M. Buck, Chem. Sci. 4, 4455 (2013)

${ }^{15}$ H. Li, B. Xu, D. Evans, and J. E. Reutt-Robey, J. Phys. Chem. C 111, 2102 (2007)

${ }^{10}$ J. O'Shea, Y. Luo, J. Schnadt, L. Patthey, H. Hillesheimer, J. Krempasky, D. Nordlund, M. Nagasono, P. Brühwiler, and N. Mårtensson, Surf. Sci. 486, 157 (2001)

${ }^{17}$ O. Adak, G. Kladnik, G. Bavdek, A. Cossaro, A. Morgante, D. Cvetko, and L. Venkataraman, Nano Lett. 15, 8316 (2015)

${ }^{18}$ A. Gibson, R. Temperton, K. Handrup, and J. O'Shea, Surf. Sci. 657, $69(2017)$ 